\title{
A common variant in Myosin-18B contributes to mathematical abilities in children with dyslexia and intraparietal sulcus variability in adults
}

\author{
KU Ludwig ${ }^{1,2}$, P Sämann ${ }^{3}$, M Alexander ${ }^{1,2}$, J Becker ${ }^{1,2}$, J Bruder $^{4}, \mathrm{~K} \mathrm{Moll}^{5,10}$, D Spieler $^{3,6}, \mathrm{M} \mathrm{Czisch}^{3}$, A Warnke ${ }^{7}$, SJ Docherty ${ }^{8}$, \\ OSP Davis ${ }^{8}$, R Plomin $^{8}$, MM Nöthen ${ }^{1,2}$, K Landerl $^{9}$, B Müller-Myhsok ${ }^{3}$, P Hoffmann ${ }^{1,2,11}$, J Schumacher ${ }^{2,11}$, G Schulte-Körne ${ }^{4,11}$ \\ and D Czamara, ${ }^{3,11}$
}

The ability to perform mathematical tasks is required in everyday life. Although heritability estimates suggest a genetic contribution, no previous study has conclusively identified a genetic risk variant for mathematical performance. Research has shown that the prevalence of mathematical disabilities is increased in children with dyslexia. We therefore correlated genomewide data of 200 German children with spelling disability, with available quantitative data on mathematic ability. Replication of the top findings in additional dyslexia samples revealed that rs 133885 was a genome-wide significant marker for mathematical abilities $\left(P_{\text {comb }}=7.71 \times 10^{-10}, n=699\right)$, with an effect size of $4.87 \%$. This association was also found in a sample from the general population $(P=0.048, n=1080)$, albeit with a lower effect size. The identified variant encodes an amino-acid substitution in MY018B, a protein with as yet unknown functions in the brain. As areas of the parietal cortex, in particular the intraparietal sulcus (IPS), are involved in numerical processing in humans, we investigated whether rs 133885 was associated with IPS morphology using structural magnetic resonance imaging data from 79 neuropsychiatrically healthy adults. Carriers of the MYO18B risk-genotype displayed a significantly lower depth of the right IPS. This validates the identified association between rs133885 and mathematical disability at the level of a specific intermediate phenotype.

Translational Psychiatry (2013) 3, e229; doi:10.1038/tp.2012.148; published online 19 February 2013

\section{Introduction}

Mathematical ability is a quantitative phenotype for which substantial heritability estimates have been reported..$^{1-3}$ Only one genome-wide association study (GWAS) of mathematical performance has been performed to date. ${ }^{4}$ This study involved individuals from the general population but failed to detect any genome-wide significant association. This may have been due to the contribution of multiple genes with low effect sizes, epistasis, gene-environment interactions or technical aspects of the applied pooling method. ${ }^{5}$ Research has shown that among children with dyslexia, which is a specific developmental disorder of learning to read or to spell, ${ }^{6}$ the prevalence of deficits in mathematical abilities is higher than in the general population. ${ }^{7,8}$ We hypothesized that if distinct cognitive processes underlying mathematical disability are preferentially affected in individuals with dyslexia, investigation of this population might limit the genetic heterogeneity, and thus increase statistical power for the identification of risk genes.
Although differing brain areas have been implicated in the performance of mathematic-related tasks across studies, ${ }^{9}$ several lines of evidence suggest that the intraparietal sulcus (IPS) has a pivotal role in the representation of numerosity. ${ }^{10,11}$ The IPS, in particular its right horizontal segment, is robustly activated during the active processing of numbers, ${ }^{12,13}$ and studies of healthy individuals have revealed that this is independent of their exact notation. ${ }^{12,14-18}$ Specific IPS responses to non-symbolic numerical processing have been demonstrated in 4-year-old children with remarkable preservation of this response pattern into adulthood. ${ }^{19}$ Abnormalities in IPS structure or function have also been detected in other disorders affecting mathematical skills, such as dyscalculia. ${ }^{20-23}$ Structural magnetic resonance imaging (MRI) has pointed out lower gray matter volume in the right IPS and bilateral cingulofrontal areas in 9-year olds with developmental dyscalculia ${ }^{22}$ and lower gray matter volume in more widespread posterior brain regions in a similar age group. ${ }^{24}$ Increased gray matter density was detected in the left

\footnotetext{
${ }^{1}$ Department of Genomics, Life and Brain Center, University of Bonn, Bonn, Germany; ${ }^{2}$ Institute of Human Genetics, University of Bonn, Bonn, Germany; ${ }^{3}$ Max Planck Institute of Psychiatry, Munich, Germany; ${ }^{4}$ Department of Child and Adolescent Psychiatry and Psychotherapy, University Hospital Munich, Munich, Germany; ${ }^{5}$ Department of Psychology, University of Salzburg, Salzburg, Austria; ${ }^{6}$ Institute of Human Genetics, Helmholtz Zentrum München-German Research Center for Environmental Health, Neuherberg, Germany; ${ }^{7}$ Department of Child and Adolescent Psychiatry and Psychotherapy, University Hospital Würzburg, Würzburg, Germany; ${ }^{8}$ King's College London, MRC Social, Genetic and Developmental Psychiatry Centre, Institute of Psychiatry, London, UK and ${ }^{9}$ Department of Psychology, University of Graz, Graz, Austria

Correspondence: Dr D Czamara, Statistical Genetics Group, Max Planck Institute of Psychiatry, Kraepelinstr. 2-10, Munich D-80804, Germany.

E-mail: darina@mpipsykl.mpg.de

${ }^{10}$ Current address: Department of Psychology, University of York, York, UK.

${ }^{11}$ These authors contributed equally to this work.

Keywords: dyscalculia; dyslexia; genomic imaging; mathematics; quantitative trait; sulcal morphology

Received 25 October 2011; revised 26 November 2012; accepted 2 Decemeber 2012
} 
IPS in 15-year-old dyscalculics with preceding preterm birth. ${ }^{23}$ Turner syndrome in adults, which is associated with specific deficits in numerical processing and visuo-spatial functions, exhibited lower depth of the right IPS with trend length and volumetric deficits. ${ }^{25}$

The aim of this study was to identify genetic variants contributing to mathematical ability (addition, multiplication and numerosity judgements (NJs)). We applied a quantitative trait design, and used genome-wide data of German children with dyslexia. We then followed-up the most significant findings in different independent samples. Finally, a structural MRI study was performed to validate our genetic findings at the level of an intermediate phenotype.

\section{Materials and methods}

The study was approved by the respective ethics committees, and written informed consent was obtained from all probands or their legal representatives. All association tests were performed using quantitative arithmetic data. Numbers presented in this section refer to sample sizes before statistical quality control. The behavioral test batteries (Supplementary Methods) and sample characteristics (Supplementary Tables 1, 2) are presented online.

\section{Individuals with dyslexia}

German sample. The German sample comprised 386 children who were subdivided into: (i) initial GWAS $(n=200$, discovery sample); and (ii) first replication sample $(n=186$, replication-1). The children were recruited at the Universities of Marburg and Würzburg in Germany. A diagnosis of dyslexia was assigned when the result of an age appropriate spelling test was at least 1 s.d. lower than expected on the basis of intelligence quotient measurements. ${ }^{26}$ In each individual, two mathematical abilities were tested (Supplementary Methods), among others. ${ }^{27}$ Briefly, arithmetic abilities were assessed using addition and multiplication tasks. The two scores were then combined, and the mean was calculated as a measure of mathematical calculation phenotype (mathematical calculation, MC). A second task addressed NJ. For NJ, subjects were asked to make judgements on the number of objects or dots presented. In a previous study, a principal component analysis revealed that $\mathrm{MC}$ and $\mathrm{NJ}$ contributed to a common 'basic mathematical ability factor' (BMAF). ${ }^{27}$ This factor was recalculated for the present data, and was used as a third quantitative phenotype.

German-Austrian replication-2 sample. A total of 206 German (Munich) and 118 Austrian (Salzburg) children were recruited within the context of the NeuroDys consortium. A diagnosis of dyslexia was assigned if reading performance was 0.75 s.d. below that expected on the basis of age and intelligence quotient. Mathematical ability was assessed by $\mathrm{MC}$ and NJ. For the genetic association analysis, MC and NJ were Z-standardized, and the mean of both phenotypes was used as a combined measure (BMAF).

\section{Non-affected samples}

German-Austrian control sample. The ascertainment process and test batteries used for the 214 controls from
Munich and the 207 controls from Austria were analogous to those used for the German-Austrian replication-2 sample. To be included as a control, the child was required to have a reading performance of 0.85 s.d. above the age norm.

The mathematical test batteries (addition, multiplication and $\mathrm{NJ}$ ) were analogous to those used for the German-Austrian replication-2 sample.

Twin Early Development Study (TEDS) sample. This sample constituted a population-based sample. Children were recruited as previously described, ${ }^{28}$ and each performed tests of reading, spelling and mathematical ability at the ages of 7, 9 and 10 years. The mathematics-related phenotypes were assessed by: (i) using and applying mathematics (including addition and subtraction tasks); (ii) number tasks (including NJ and counting); and (iii) the perception of shapes, space and measures. All data were ascertained according to an ordinal scale. To generate a quantitative phenotype, standardized scores were summed and re-standardized. Given the genetic continuity of quantitative traits, ${ }^{2}$ an average of this score across the three age groups was used. In the present analyses, 1081 unrelated individuals with measurements at all three time points were randomly chosen (one from each twin pair). This sample comprised 356 individuals who had been included in the previous study by Docherty et al. ${ }^{4}$ However, as rs133885 was not one of the individually genotyped markers used in that study, this sample can be considered an independent replication sample.

Genotyping. The genome-wide data were retrieved from a previous GWAS on dyslexia that had been conducted using the Illumina HumanHap300k BeadChips (Illumina, San Diego, CA, USA). In the replication studies, singlenucleotide polymorphisms (SNPs) for the single-marker and haplotype analyses were genotyped using the Sequenom MassARRAY iPlex Gold system (Sequenom, San Diego, CA,USA) and the SpectroTYPER software v3.1 (Sequenom).

Statistical analysis. For the GWAS data, analysis of all autosomal SNPs was performed using analysis of variance, the WG-Permer software (http://www.wg-permer.org/), and $\mathrm{R},{ }^{29}$ as previously described. ${ }^{30}$ Genome-wide significance was defined according to Dudbridge and Gusnanto $\left(P<7.2 \times 10^{-08}\right) .^{31}$ The quality parameters used in the replication samples and the GWAS sample were identical. Quantitative association analyses of the replication and combined samples were also conducted with $\mathrm{R}$ (using the glm option in the R-library stats). The covariates were: (i) sample origin (replication-2); and (ii) sample origin, spelling and word reading (combined samples).

Haplotypic effects were first tested in the genome-wide data, using a sliding window approach for up to five SNPS (including rs133885). Nominal significant haplotypes were reanalyzed in the replication-1 sample. Haplotypic $P$-values and linkage disequilibrium measures were calculated using PLINK. ${ }^{32}$ Power analysis was performed post hoc, using the Genetics Power Calculator ${ }^{33}$ and the settings 'no dominance', 'singletons with no parental genotypes' and a genotypic frequency of 0.317 (corresponding to the GG genotype in CEU HapMap-individuals). Effect sizes were estimated using 
the $R^{2}$-goodness-of-fit measure and two settings: (i) the $R^{2}$ for each subsample; and (ii) the weighted average of $R^{2}$ across both dyslexia- and non-affected samples, respectively (Supplementary Table 3).

Sequencing analysis. Sequencing of the first four exons of MYO18B long was performed exon-wise, in 35 children (replication-1 sample), according to standard procedures. Briefly, PCR products were purified using Agencourt AMPure (Beckman Coulter, Krefeld, Germany) and sequenced in both directions. Sequencing products were purified by Agencourt CleanSEQ (Beckman Coulter, Krefeld, Germany) and run on an ABI Capillary Sequencer (3130XL, Applied Biosystems, Darmstadt, Germany). Data were analyzed using ChromasLite 2.0 (http://technelysium.com.au/). Detected variants were followed-up in the entire discovery sample. Primer sequences and protocols are available on request.

Expression analysis. Expression analysis for $M Y 018 B$ was performed using $\mathrm{N}$-terminal primers for $M Y O 18 B^{\text {long }}$, and primers for the core region of $M Y O 18 B$ (overlapping in

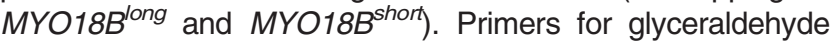
3-phosphate dehydrogenase were used as housekeeping controls. Complementary DNAs were obtained from customary multiple tissue complementary DNA panels (Human MTC-panel Human I (Lot-Nr.7080213); and Fetal (Lot-Nr. 7060067, all Clontech/Takara Bioscience, Saint-Germain-enLaye, France)). PCR products were assessed on a $1 \%$ agarose gel. MYO18B identity was confirmed by sequencing. Primer sequences and protocols are available on request.

Structural MRI analysis. A structural MRI study was performed to measure sulcal depth in the IPS. This involved

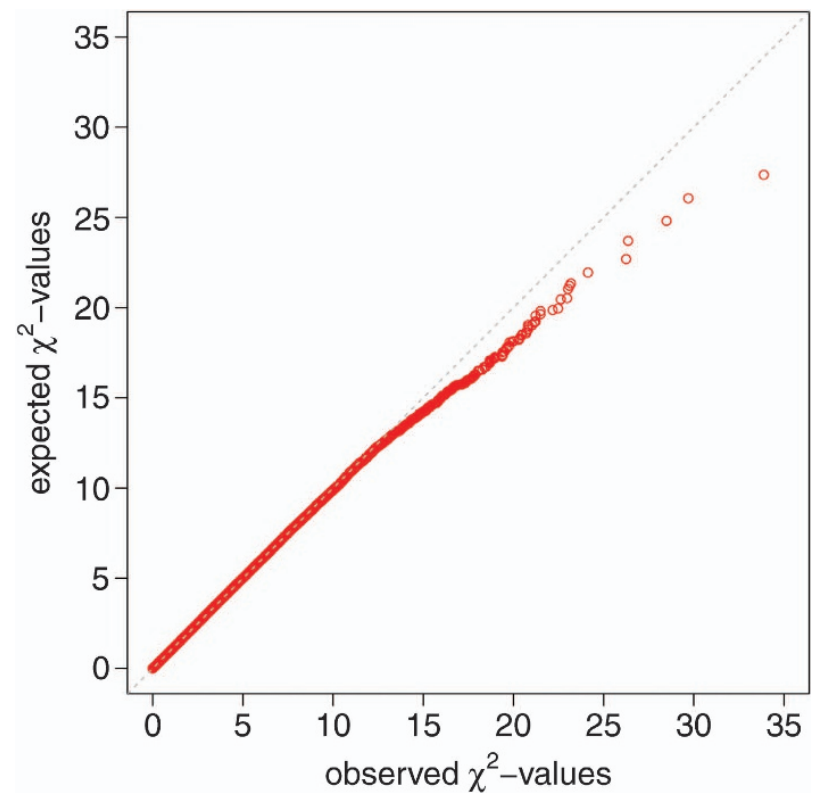

Figure 1 Quantile-Quantile $(Q-Q)$ plot for the basic mathematical ability factor (BMAF). Q-Q plot of the GWAS of 200 German dyslexia patients: the distribution of expected (under the null-hypothesis) and observed $\chi^{2}$ values for the genotypic model are depicted. Adherence to the diagonal, which is almost perfect in the lower parts of the distribution, indicates no inflation of the statistics. automated sulcus allocation and a feature extraction pipeline. Voxel-based morphometry (VBM) was used to explore genotype-dependent differences in regional gray matter volume. The genotypic and imaging data were retrieved from an independent genetic study ${ }^{34}$ of 100 neuropsychiatrically healthy adult subjects. All details of subject characteristics, MRI sequence parameters, image postprocessing steps and the software used for sulcus morphology analysis and VBM are provided online (Supplementary Methods, Supplementary Table 4). Briefly, sulcus morphology analysis was directed to the bilateral IPS that contains the horizontal segment (subsequently referred to as IPS main $_{\text {). The bilateral }}$ central sulcus was chosen as a control structure. The average sulcus depth and the total sulcus volume of all classified sulci in each hemisphere were calculated for later use as covariates. Statistical analysis was performed using analysis of covariance, and the covariates age, gender, and the average sulcus depth or the total sulcus volume. Post hoc, the sulcus measures area surface and length were analyzed for the right IPS $S_{\text {main }}$ only to complete the morphometric characterization. After optimal intersubject co-registration, whole-brain VBM was performed in the same sample using the conservative cluster-based statistical inference method.

\section{Results}

\section{Genetic studies}

Initial GWAS. A Q-Q plot for the BMAF analysis is shown in Figure 1 (for MC and NJ, see Supplementary Figure 1). No significant deviation was observed. One SNP (rs133885) reached genome-wide significance with BMAF, and two SNPs (rs1399428 and rs4837521) attained this level with NJ, based on $P<7.2 \times 10^{-08}$ (Table 1, Figure 2, Supplementary Figure 2). None of these three markers showed significant association with dyslexia.

Replication in dyslexia samples. In the replication-1 sample, association between rs133885 and BMAF was confirmed ( $P=0.0049)$, but replication failed for the two other SNPs (Table 1). In the combined German sample, the association for rs133885 was strongest in the dominant/recessive model, with carriers of the rs133885 GG-genotype showing weaker performance in mathematical tasks than AA/AG carriers $\left(P=8.81 \times 10^{-10}\right.$, carrier-A model, Supplementary Figure 3 ). Calculation of the fraction of BMAF variance that was accounted for by rs 133885 yielded an estimated effect size of $9.4 \%$. Haplotype analyses did not improve $P$-values for the association with BMAF, compared with rs133885 alone (Supplementary Table 5).

Genotyping rs133885 in the dyslexia replication-2 sample revealed a significant BMAF association $(P=0.0446)$. Combining all cases resulted in a $P$-value of $7.71 \times 10^{-10}$. At least a trend toward association was found for $M C$ and $\mathrm{NJ}$, but neither of these two measures was solely responsible for the association (Supplementary Table 6). As some of the dyslexia cases showed very low values for BMAF, an inverse rankbased transformation was performed to achieve a more normal distribution. Thereafter, the $P$-value remained genome-wide significant $\left(P=8.43 \times 10^{-10}\right.$, Supplementary Table 7$)$. The overall effect size for the association between 
Table 1 SNPs with genome-wide significance $\left(P<7.2 \times 10^{-08}\right)$ in the GWAS sample

\begin{tabular}{|c|c|c|c|c|c|c|c|}
\hline \multicolumn{4}{|c|}{ Marker information } & \multirow{2}{*}{$\begin{array}{l}\text { Statistical } \\
\text { model }^{a}\end{array}$} & \multicolumn{3}{|c|}{ P-values German sample ${ }^{b}$} \\
\hline SNP (phenotype) & Chr & $\begin{array}{c}\text { Position } \\
(d b S N P 131)\end{array}$ & $\begin{array}{l}\text { Gene } \\
\text { (hg19) }\end{array}$ & & $\begin{array}{l}\text { Discovery- } \\
\text { sample }\end{array}$ & $\begin{array}{c}\text { Replication-1 } \\
\text { sample }\end{array}$ & $\begin{array}{c}\text { German sample } \\
\text { combined }\end{array}$ \\
\hline rs1399428 (NJ) & 9 & 120660568 & NA & $\begin{array}{l}\text { genotypic } \\
\text { het./hom. }\end{array}$ & $\begin{array}{l}2.40 \times 10^{-08} \\
3.19 \times 10^{-09}\end{array}$ & $\begin{array}{l}0.2335 \\
0.1270\end{array}$ & $\begin{array}{l}7.01 \times 10^{-06} \\
1.09 \times 10^{-06}\end{array}$ \\
\hline rs4837521 (NJ) & 9 & 120654461 & NA & $\begin{array}{l}\text { genotypic } \\
\text { het./hom. }\end{array}$ & $\begin{array}{l}1.53 \times 10^{-07} \\
2.03 \times 10^{-08}\end{array}$ & $\begin{array}{l}0.0498 \\
0.0201^{c}\end{array}$ & $\begin{array}{l}5.67 \times 10^{-07} \\
8.46 \times 10^{-08}\end{array}$ \\
\hline rs133885 (BMAF) & 22 & 26159289 & MYO18B & $\begin{array}{l}\text { genotypic } \\
\text { allelic } \\
\text { carrier-A }\end{array}$ & $\begin{array}{l}4.39 \times 10^{-08} \\
1.24 \times 10^{-06} \\
5.79 \times 10^{-09}\end{array}$ & $\begin{array}{l}0.0045 \\
0.0012 \\
0.0049\end{array}$ & $\begin{array}{l}2.75 \times 10^{-09} \\
9.83 \times 10^{-09} \\
8.81 \times 10^{-10}\end{array}$ \\
\hline
\end{tabular}

Abbreviations: BMAF, basic mathematical ability factor; GWAS, genome-wide association study; het., heterozygous; hom., homozygous; NA, not assigned; NJ, numerosity judgement; SNP, single-nucleotide polymorphism.

${ }^{a}$ Genetic models with a $P$-value $<10^{-05}$ for the specific phenotype (discovery-sample). ${ }^{b} P$-values in bold if genome-wide significant (discovery-sample), or $P<0.05$ (replication-1 sample). ${ }^{\mathrm{C}}$ Risk alleles not identical between discovery and replication-1 sample.

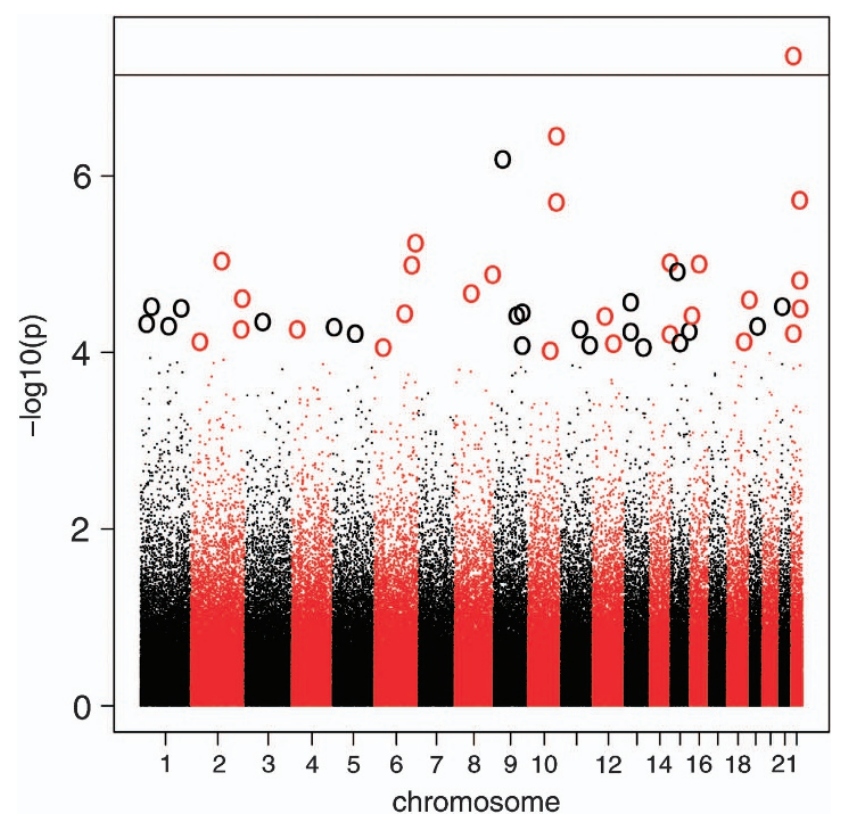

Figure 2 Genome-wide results for the basic mathematical ability factor (BMAF). Association results of the GWAS of 200 German dyslexia patients for the genotypic model. Genomic position along the 22 autosomes is represented on the $x$ axis; $-\log 10(P$-value $)$ is represented on the y axis. SNPs with a nominal $P$-value below $10^{-04}$ are depicted as circles. The horizontal line indicates the threshold for genomewide significance $\left(P<7.2 \times 10^{-08}\right)$.

rs133885 and BMAF in samples with dyslexia was $4.87 \%$ (Table 2).

Replication independent of dyslexia. Genotyping rs133885 in the population-based TEDS sample revealed a significant $P$-value of 0.048 (Table 2). As the phenotypes 'numbers' and 'using and applying mathematics' are the most comparable to BMAF, we recalculated the association for TEDS after removing the phenotype 'shapes, space, and measures'. The $P$-value remained stable $(P=0.041$; Supplementary Table 8). The rs133885 association in the German-Austrian control sample was in the same direction, although it did not reach significance $(P=0.486$, Table 2$)$.
Sequencing analysis. The SNP rs133885 maps to the coding region of the Myosin-18B gene (MYO18B, MIM607295). Two distinct MYO18B protein isoforms have been identified: MYO18B ${ }^{\text {long }}$ (2567 amino acids, SwissProt accession number Q8IUG5-1); and MYO18B ${ }^{\text {short }}(2080$ amino acids, Q8IUG5-2). The p.E44G substitution mediated by rs 133885 is located in the $\mathrm{N}$-terminus specific to $\mathrm{MYO} \mathrm{B}^{\mathrm{long}}$. Sequencing of this $\mathrm{N}$-terminal region revealed the presence of three common variants, two of which (rs61734946 and rs13058434) were non-synonymous. In the discovery sample, rs61734946 was strongly associated with mathematical abilities, but this effect was driven by rs133885 (Supplementary Table 9). The SNP rs13058434 (p.P177L) showed nominally significant association with BMAF in the subgroup of children who were not-at-risk according to rs133885 genotype.

Expression analysis. Glyceraldehyde 3-phosphate dehydrogenase expression was found in all analyzed tissues and stages. MYO18B long was expressed in all tissues except brain and adult lung, and strong expression was found in skeletal muscle and heart. The core part of MYO18B was abundant in all tissues, including adult brain. However, this expression was not observed in the developing fetal brain (Supplementary Figure 4).

Structural MRI analysis. The results of the sulcus analysis on 79 individuals are shown in Figure 3. In carriers of the rs133885 GG-genotype, a lower average depth of the $I_{P S}$ main (mean \pm s.d. $23.18 \pm 2.62$ versus $24.70 \pm 2.05 \mathrm{~mm}$; $F_{1,74}=11.74, P=0.001$ ) was observed after adjustment for age, gender and average right-hemispheric sulcus depth. Similar results were obtained for right IPS sulcus volume $\left(1665 \pm 535\right.$ versus $1900 \pm 425 \mathrm{~mm}^{3} ; F_{1,74}=4.69, P=0.033$; see Supplementary Results for surface area and sulcus length). In the left hemisphere, genotype effects were in the same direction, with a trend level for IPS depth $(24.79 \pm 2.15$ versus $\left.25.41 \pm 2.14 \mathrm{~mm} ; F_{1,74}=3.69, P=0.059\right)$; but nonsignificant for sulcus volume $(P=0.167)$. No genotype effect was found for the depth or volume of the central sulcus in either hemisphere $(P>0.4$; Figure 3$)$. 
Table 2 Association analyses for rs 133885 in dyslexia, control and population-based samples

\begin{tabular}{|c|c|c|c|c|c|c|c|}
\hline \multirow[t]{2}{*}{ Sample } & \multirow{2}{*}{$\begin{array}{l}\text { Total number } \\
\text { of individuals }^{a}\end{array}$} & \multicolumn{2}{|c|}{ Genotypes $A A / A G$} & \multicolumn{2}{|c|}{ Genotype GG } & \multirow[t]{2}{*}{$\mathrm{P}$-value $e^{b}$} & \multirow[t]{2}{*}{ Effect size } \\
\hline & & $\begin{array}{c}\mathrm{N} \\
\text { (Frequency) }\end{array}$ & $\begin{array}{c}B M A F \\
\text { mean } \pm \text { s.d. }\end{array}$ & $\begin{array}{c}\mathrm{N} \\
\text { (Frequency) }\end{array}$ & $\begin{array}{c}B M A F \\
\text { mean } \pm \text { s.d. }\end{array}$ & & \\
\hline Discovery & 200 & $132(0.66)$ & $-0.31 \pm 1.55$ & $68(0.34)$ & $-2.10 \pm 2.61$ & $5.78 \times 10^{-09}$ & $15.78 \%$ \\
\hline Replication-1 & 183 & $126(0.69)$ & $-0.11 \pm 1.90$ & $57(0.31)$ & $-0.89 \pm 1.80$ & $4.91 \times 10^{-03}$ & $3.63 \%$ \\
\hline Replication-2 & 316 & $229(0.72)$ & $-0.39 \pm 0.66$ & $87(0.28)$ & $-0.55 \pm 0.80$ & $4.46 \times 10^{-02}$ & $0.88 \%$ \\
\hline All case samples ${ }^{\mathrm{d}}$ & 699 & - & - & - & - & $7.71 \times 10^{-10}$ & $4.87 \%$ \\
\hline German-Austrian controls & 391 & $275(0.70)$ & $0.42 \pm 0.77$ & $116(0.30)$ & $0.41 \pm 0.81$ & $4.86 \times 10^{-01}$ & $0.01 \%$ \\
\hline UK TEDS & 1080 & $741(0.69)$ & $0.10 \pm 0.85$ & $339(0.31)$ & $0.01 \pm 0.79$ & $4.82 \times 10^{-02}$ & $0.26 \%$ \\
\hline $\begin{array}{l}\text { All control/ population } \\
\text { samples }^{d}\end{array}$ & 1471 & - & - & - & - & $7.52 \times 10^{-02}$ & $0.007 \%$ \\
\hline
\end{tabular}

Abbreviations: BMAF, basic mathematical ability factor, as defined in the respective samples; $N$, number of observations; TEDS, Twin Early Development Study. ${ }^{a}$ After quality control. No deviation from Hardy-Weinberg was observed for any of the samples. Demographic details of the samples are presented as Supplementary Material online. ${ }^{b}$ One-sided, except for the German discovery and combined samples (carrier-A model). Sample origin was used as a covariate in replication-2. ${ }^{c}$ Measures based on $R^{2}$-goodness-of-fit coefficient. In the presence of covariates, effect size was based on the difference between the $R^{2}$-coefficient in the full model, and the $R^{2}$-coefficient including only the covariates in the regression model. ${ }^{\mathrm{d}}$ Analysis of combined cases and controls involved use of sample origin, spelling and word reading as covariates.

a
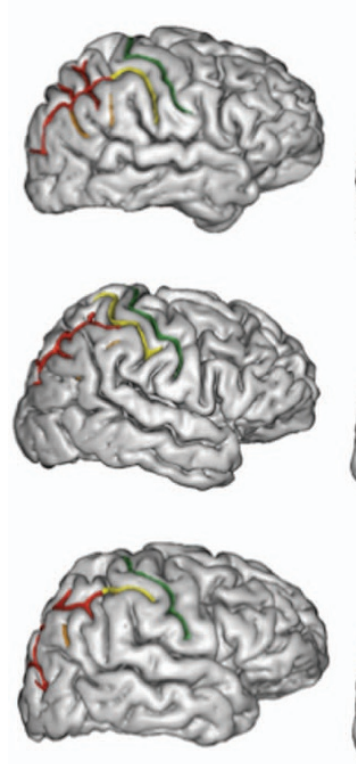

Intraparietal sulcus (IPS ${ }_{\text {main }}$ )

Inferior postcentral sulcus

Additional IPS branches

Central sulcus b

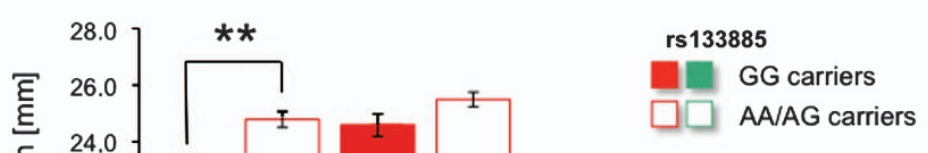

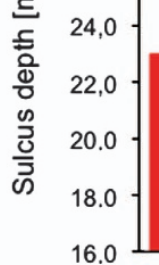
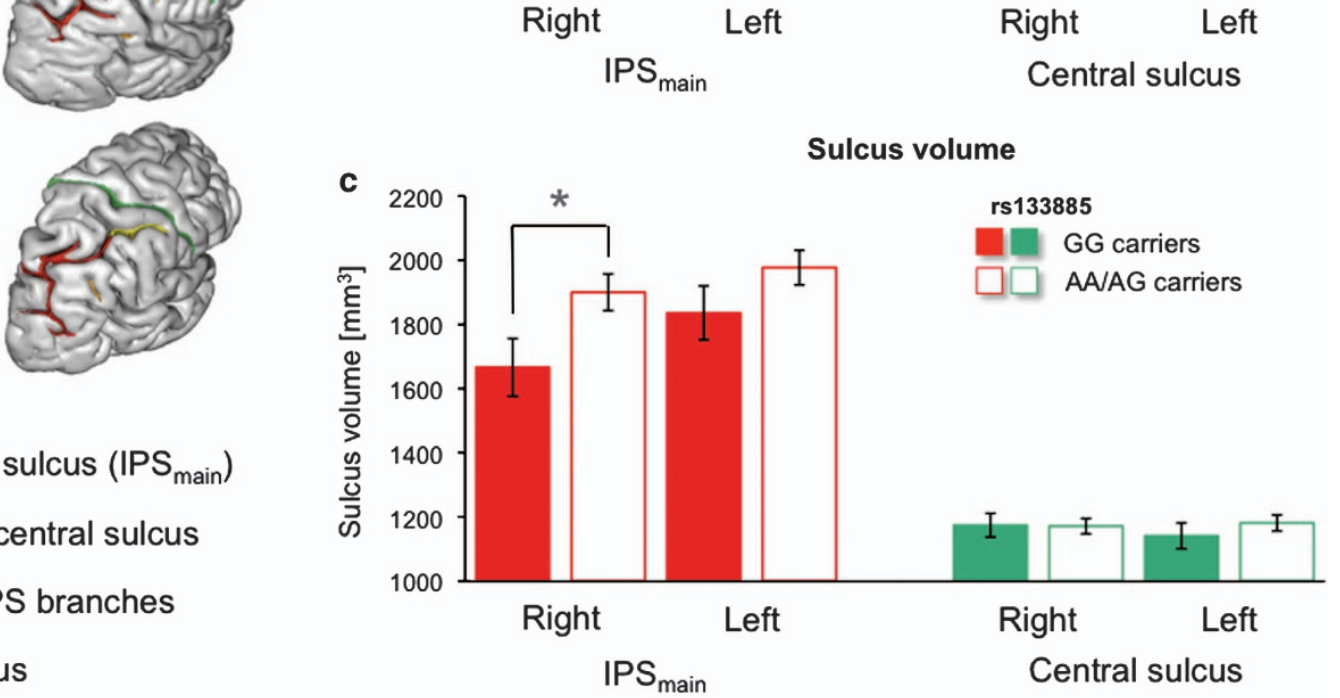

Figure 3 Morphological analysis of the intraparietal sulcus (IPS). (a) Automated assignment was performed for right IPS segmentations and central sulcus. Three representative cases are shown. Note inter-individual variance of IPS geometry. Structural measurements were made for the depth (b) and volume (c) of the hypothesized sulcus and a control sulcus (central sulcus). Analysis of covariance comparing A-allele carriers with non-A-allele carriers was performed for both measures, and was corrected for age, gender, and hemisphere averages for sulcal depth and volume, respectively. Bars show estimated means and 1 s.e.m. ${ }^{*} P<0.05$; ${ }^{* \star} P<0.0063$ (Bonferroni-corrected threshold). 
The effect on the depth of the right IPS main $_{\text {withstood }}$ correction for multiple testing $\left(P_{\text {corrected }}=0.008\right)$. Regional specificity was emphasized by an explorative analysis of all extracted sulci, which yielded no stronger association than

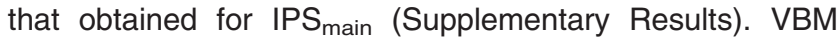
analysis revealed no significant genotype effect on regional gray- or white matter volume (Supplementary Results).

\section{Discussion}

In this study, we systematically investigated genetic variants contributing to mathematical abilities. Genomewide data were retrieved from a GWAS of dyslexia, in view of previous reports that the prevalence of mathematical deficits is higher in dyslexia compared with the general population. ${ }^{7,8}$ Phenotypic data on mathematical performance were available for all children, thus enabling a quantitative approach.

Although three variants were genome-wide significant, only rs133885 was successfully replicated. The other two SNPs are located on chromosome 9q33.1 (rs1399428 and rs4837521) and showed genome-wide significance with NJ. They are around $6.1 \mathrm{~kb}$ apart and share high $\operatorname{LD}\left(r^{2}=0.71\right.$, $\left.\mathrm{D}^{\prime}=0.88\right)$, indicating that they reflect the same effect. Unexpectedly, however, we failed to replicate this association in the replication-1 sample, which may be due to a number of reasons. First, children included in the GWAS were more severely affected with dyslexia compared with children in the replication-1 sample. It is therefore possible that the genetic effect of the $9 q 33.1$ locus on mathematical ability is triggered by the severity of spelling impairment. A second possible explanation is that the two markers appeared among the top GWAS hits simply by chance, without a true contribution to the quantitative trait analyzed.

It also has to be mentioned that it is highly possible that genetic components mainly defining $\mathrm{NJ}$ or MC may exist. By restricting our replication study to the three top hits, we might have missed other possible causal variants. Further experiments are therefore warranted to dissect the contribution of the $9 q 33.1$ locus and of other yet unknown loci to arithmetic abilities.

rs133885 was replicated with an estimated effect size of $9.4 \%$ in the German sample. Although this might be an overestimate because of a 'winner's curse' effect in the GWAS data, the observation of $3.6 \%$ in the replication-1 sample independently supports a strong contribution of rs133885 in individuals with spelling disorder. The difference in effect sizes, however, may also be attributable to the different degrees of dyslexia severity in the samples. Results of a stratification analysis in the German sample support this hypothesis, as an increasing effect size was observed with increasing degree of spelling disorder (Supplementary Table 10). An even lower effect size was observed in the replication-2 sample. Although this may have resulted from simple power issues, this observation might also reflect potential differing underlying core deficits in children with spelling disorder (initial, replication-1), and those with a disorder in reading (replication-2).

Although different phenotypic measures were used for the assessment of the complex construct of mathematical abilities, a consistent pattern of findings emerged from our data: the genetic association appeared to be equally strong for MCs and NJ. Our measures of mathematical abilities (accuracy and fluency of mental addition and multiplication) are influenced by verbal as well as general educational deficits that are typically experienced by dyslexic individuals and the genetic association might reflect these particular problems. However, this is not the case for the very simple task of number comparison. It has been claimed that arithmetical abilities are built on an inherited core knowledge system that can be assessed by such simple tasks ${ }^{35-37}$ suggesting that our findings are a direct indication for the genetic basis of this numerical core knowledge, rather than mathematical ability as such.

The aforementioned results suggest that rs133885 contributes to mathematical performance in children with dyslexia, and that a more pronounced effect is found in those with spelling disorder. On the basis of this, we investigated whether rs133885 was associated with mathematical performance independent of weak reading or spelling disabilities. Although we observed a nominally significant association in the population-based sample, no statistically significant association was observed in children with superior reading performance (unfortunately, no sample showing superior spelling performance was available). However, the observation of a trend in the hypothesized direction suggests that an effect of rs133885 might be detected in larger samples (Supplementary Table 3). We also attempted a subgroup analysis of the TEDS, involving only those individuals with a weak performance in both the mathematical and the readingspelling tasks. However, only a few individuals met these criteria, and so this analysis could not be completed (data not shown). In total, our results give rise to the hypothesis that the association between rs133885 and mathematical abilities is enhanced in dyslexic individuals. One possible explanation therefore might be the lack of compensatory mechanisms in dyslexic individuals, which render the genetic effect more pronounced at phenotypic level and, thus, more likely to be detected in smaller samples.

The associated variant rs133885 encodes an amino-acid within MYO18B ${ }^{\text {long }}$ (p.E44G). MYO18B belongs to the family of unconventional myosins, ${ }^{38}$ and has been implicated in carcinogenesis ${ }^{39,40}$ and the composition of myofibrillar structures. ${ }^{41}$ The functionally relevant position of rs133885 suggests that the variant is causative, and this was supported by the results of the haplotype analyses. Based on recent evidence suggesting the presence of important actin-binding sites within the MYO18B N-terminus ${ }^{42,43}$ (Supplementary Figure 5, Supplementary Results), we hypothesized that other sequence alterations of the $\mathrm{N}$-terminus might also affect mathematical performance. Although no novel variants were detected, the independent association of a second common non-synonymous variant p.P177L suggests that additional $\mathrm{N}$-terminal alterations within $M Y O 18 B^{\text {long }}$ may contribute to mathematical abilities.

The functional consequences of rs133885 are difficult to predict at the time of writing. The expression of both MYO18B isoforms in skeletal muscle and heart is consistent with the reported function of MYO18B in myocardial structures. ${ }^{41}$ Although substantial $M Y O 18 B^{\text {short }}$-expression was 
observed in the adult brain, no evidence was obtained for MYO18B-expression in the developing fetal brain. However, as the fetal customary complementary DNA panel comprised tissues from 20 to 30 weeks old fetuses, the relevant developmental time point might have been missed. If MYO18B is expressed at other developmental time points, this might explain the failure to detect MYO18B expression in the fetal samples. Interestingly, one previous study demonstrated a contribution of MYO18B to cognitive phenotypes. ${ }^{44,45}$ In one of the largest GWAS case-control studies of schizophrenia performed to date, ${ }^{45}$ a variant located in the first exon of $M_{Y O 18 B^{l o n g}}$ was among the most strongly associated SNPs (rs5761163). Analysis of this variant in our GWAS data revealed an association with BMAF $(P=0.00546)$, although this effect disappeared when the analysis was conditioned on rs133885 $(P=0.19)$.

On the functional level, the sulcus analysis of the IPS demonstrated that carriers of the associated rs133885 GG-genotype showed a lower average depth of the right $I P S_{\text {main }}$. The identification of this intermediate phenotype is well concordant with the fact that basic features of gyrogenesis and sulcal morphology are under tight genetic control. ${ }^{46,47}$

The lack of differences in gray matter and white matter volume as measured by VBM is divergent from previously reported VBM comparisons between dyscalculic subjects and respective age-matched healthy controls. In 9-year-old children with developmental dyscalculia, Rotzer et al. ${ }^{22}$ found reduced gray matter volume in the right IPS and cingulofrontal areas and Rykhlevskaia et al., ${ }^{24}$ in the same age class, found reduced gray matter volume in more widespread occipital, cerebellar and posterior parietal regions. Isaacs et al. ${ }^{23}$ revealed increased gray matter density, a measure sensitive to mesoscopic gyral differences, in the left parietal lobe in dyscalculic adolescents with a history of preterm birth. Several points may underlie the divergence between these results and our result: mainly, these reports studied subjects with a clinical manifestation of dyscalculia whereas the variance of the continuous behavioral phenotype explained by the genotype in this study ranged below $10 \%$. This suggests that also at the brain level lower genotypedependent effects may be expected compared with alterations in subjects that have impairments classifiable as dyscalculia. Although we have directed our analysis to the IPS based on converging evidence for this area being key to the representation of numerosity, formally, our MRI analysis was explorative as no generally accepted structural or functional imaging endophenotypes of arithmetic abilities (or dyscalculia) have been defined yet. Recent GWAS imaging studies, however, point out, that even for validated imaging endophenotypes such as hippocampal volume, the strongest detectable genetic univariate effects are not necessarily stronger than typical associations with a neuropsychiatric phenotype. ${ }^{48}$ A second reason for not detecting VBM associations may lie in the age discrepancy between our $\mathrm{MRI}$ sample and previous VBM reports of dyscalculia. This latter assumption is strengthened by the close analogies between our results and the report of Molko et al. ${ }^{25}$ who performed a multimodal study on adult Turner syndrome patients with dyscalculia, finding right-emphasized IPS structural differences in the sulcal analyses but only marginal gray matter alterations. Presumably, among the structural techniques, sulcus measurements could be more sensitive to gyral folding differences than VBM, which is not a surface-based technique yet, except for Molko et al., ${ }^{25}$ no combined sulcus/VBM analyses have been reported to allow for in-depth cross-correlation of these techniques.

Although involvement of the IPS in numerical processing has been well established, ${ }^{10}$ recent evidence suggests that other brain areas are also involved, ${ }^{9}$ depending on task specificities such as the type of calculation (accurate versus approximate) and type of number representation (symbolic versus non-symbolic). To determine whether our findings were specific to the IPS, we expanded our sulcal analysis to 58 sulci from each hemisphere. In the right hemisphere, one structure (superior temporal sulcus) showed nominal significance. In the left hemisphere, six additional sulci showed nominal significance with no particular regional specificity for the IPS. Despite the lack of behavioral data to demonstrate a direct association between sulcus measures and arithmetic abilities in this same study, MRI findings support the hypothesis of a specific association between rs133885 and variability in the structure of the right IPS, with weaker left-hemispheric associations possibly related to the symbolic representation of numbers in the original arithmetic tests. ${ }^{9}$ Although there is evidence from functional studies that nonsymbolic numerical processing is represented in the IPS as early as at age 4 with continuity into adulthood, ${ }^{19}$ a direct extrapolation of our structural results to young age is hampered by limited knowledge on the trajectories of the here studied sulcus measures over the life span in humans. Hence, although the here reported sulcal variations represent a plausible first neural correlate, further structural cortex measures and functional MRI activation studies on younger samples with genetic and phenotypic characterization are essential to understand which dimensions of arithmetic operations are impacted by rs 133885 .

In summary, this study identified a non-synonymous variant in $M Y O 18 B$ as the first genome-wide significant marker to contribute to human mathematical ability. Our data provide evidence that weak spelling ability might be a modifying factor for the strength of the observed association with rs133885. The association was also present in a sample from the general population, albeit with a smaller effect size. At the level of an intermediate brain phenotype, we investigated the association between rs133885 and structural variability of the IPS, which is a key structure in terms of numerical processing. We found that healthy carriers of the $M Y O 18 B$ risk-genotype exhibited reduced depth of the right IPS, thus implicating $M Y O 18 B$ in cognitive processes. Future studies should elucidate how genetic variation in this gene exactly relates to human mathematical performance.

\section{Conflict of interest}

The authors declare no conflict of interest.

Acknowledgements. We thank the children and their families for participating in the study. GSK, BMM and MMN were supported by the Deutsche Forschungsgemeinschaft, and received funding from the EU, within the context of 
the Sixth Framework Program LifeScienceHealth project 'Dyslexia genes and neurobiological pathways' (Neurodys, 018696). MMN received support for this work from the Alfried Krupp von Bohlen und Halbach-Stiftung, JS was supported by a $\mathrm{NIH} / \mathrm{DFG}$ Research Career Transition Award. TEDS is funded by the UK Medical Research Council (G500079). We are grateful to Anna Olyinyk for her support with the image quality control of the MRI sample, and Denis Riviere for his support in the employment of the BrainVisa(BV)/Anatomist software.

1. Alarcon M, DeFries JC, Light JG, Pennington BF. A twin study of mathematics disability. J Learn Disabil 1997; 30: 617-623.

2. Haworth CM, Kovas $\mathrm{Y}$, Petrill SA, Plomin R. Developmental origins of low mathematics performance and normal variation in twins from 7 to 9 years. Twin Res Hum Genet 2007; 10: 106-117.

3. Knopik VS, DeFries JC. Etiology of covariation between reading and mathematics performance: a twin study. Twin Res 1999; 2: 226-234.

4. Docherty SJ, Davis OS, Kovas Y, Meaburn EL, Dale PS, Petrill SA et al. A genome-wide association study identifies multiple loci associated with mathematics ability and disability. Genes Brain Behav 2010; 9: 234-247.

5. Pearson JV, Huentelman MJ, Halperin RF, Tembe WD, Melquist S, Homer $\mathrm{N}$ et al. Identification of the genetic basis for complex disorders by use of pooling-based genomewide single-nucleotide-polymorphism association studies. Am J Hum Genet 2007; 80: $126-139$.

6. WHO The ICD-10 Classification of Mental and Behavioural Disorders: Diagnostic Criteria for Research. World Health Organization: Geneva, 1993.

7. Dirks E, Spyer G, van Lieshout EC, de SL. Prevalence of combined reading and arithmetic disabilities. J Learn Disabil 2008; 41: 460-473.

8. Landerl K, Moll K. Comorbidity of learning disorders: prevalence and familial transmission. $J$ Child Psychol Psychiatry 2010; 51: 287-294.

9. Kaufmann L, Wood G, Rubinsten O, Henik A. Meta-analyses of developmental fMRI studies investigating typical and atypical trajectories of number processing and calculation. Dev Neuropsychol 2011; 36: 763-787.

10. Butterworth B, Varma S, Laurillard D. Dyscalculia: from brain to education. Science 2011; 332: 1049-1053.

11. Dehaene $S$, Piazza M, Pinel $P$, Cohen $L$. Three parietal circuits for number processing. Cogn Neuropsychol 2003; 20: 487-506.

12. Chochon F, Cohen L, van de Moortele PF, Dehaene S. Differential contributions of the left and right inferior parietal lobules to number processing. J Cogn Neurosci 1999; 11: $617-630$.

13. Fias W, Lammertyn J, Caessens B, Orban GA. Processing of abstract ordinal knowledge in the horizontal segment of the intraparietal sulcus. J Neurosci 2007; 27: 8952-8956.

14. Dehaene S, Spelke E, Pinel P, Stanescu R, Tsivkin S. Sources of mathematical thinking: behavioral and brain-imaging evidence. Science 1999; 284: 970-974.

15. Gruber $\mathrm{O}$, Indefrey $\mathrm{P}$, Steinmetz $\mathrm{H}$, Kleinschmidt $\mathrm{A}$. Dissociating neural correlates of cognitive components in mental calculation. Cereb Cortex 2001; 11: 350-359.

16. Naccache L, Blandin E, Dehaene S. Unconscious masked priming depends on temporal attention. Psychol Sci 2002; 13: 416-424.

17. Pesenti M, Thioux M, Seron X, De VA. Neuroanatomical substrates of arabic number processing, numerical comparison, and simple addition: a PET study. J Cogn Neurosci 2000; 12: 461-479.

18. Pinel $P$, Dehaene $S$, Riviere $D$, LeBihan $D$. Modulation of parietal activation by semantic distance in a number comparison task. Neuroimage 2001; 14: 1013-1026.

19. Cantlon JF, Brannon EM, Carter EJ, Pelphrey KA. Functional imaging of numerical processing in adults and 4-y-old children. PLOS Biol 2006; 4: e125.

20. Kucian K, Loenneker T, Dietrich T, Dosch M, Martin E, von AM. Impaired neural networks for approximate calculation in dyscalculic children: a functional MRI study. Behav Brain Funct 2006; 2: 31

21. Price GR, Holloway I, Rasanen P, Vesterinen M, Ansari D. Impaired parietal magnitude processing in developmental dyscalculia. Curr Biol 2007; 17: R1042-R1043.

22. Rotzer S, Kucian K, Martin E, von AM, Klaver P, Loenneker T. Optimized voxel-based morphometry in children with developmental dyscalculia. Neuroimage 2008; 39: 417-422.

23. Isaacs EB, Edmonds CJ, Lucas A, Gadian DG. Calculation difficulties in children of very low birthweight: a neural correlate. Brain 2001; 124(Pt 9): 1701-1707.

24. Rykhlevskaia E, Uddin LQ, Kondos L, Menon V. Neuroanatomical correlates of developmental dyscalculia: combined evidence from morphometry and tractography. Front Hum Neurosci 2009; 3: 51.
25. Molko N, Cachia A, Riviere D, Mangin JF, Bruandet M, Le BD et al. Functional and structural alterations of the intraparietal sulcus in a developmental dyscalculia of genetic origin. Neuron 2003; 40: 847-858.

26. Schulte-Körne G, Deimel W, Remschmidt H. Diagnosis of reading and spelling disorder. Zeitschritt für Kinder- und Jugendpsychiatrie und Psychotherapie 2001; 29: 113-116.

27. Schulte-Körne G, Ziegler A, Deimel W, Schumacher J, Plume E, Bachmann C et al. Interrelationship and familiality of dyslexia related quantitative measures. Ann Hum Genet 2007; 71: 160-175.

28. Oliver BR, Plomin R. Twins' Early Development Study (TEDS): a multivariate, longitudinal genetic investigation of language, cognition and behavior problems from childhood through adolescence. Twin Res Hum Genet 2007; 10: 96-105.

29. R-Development-Core-Team R: A Language and Environment for Statistical Computing. R Foundation for Statistical Computing: Vienna, Austria, 2009.

30. Roeske D, Ludwig K, Neuhoff N, Becker J, Bartling J, Bruder J et al. First genome-wide association scan on neurophysiological endophenotypes points to trans-regulation effects on SLC2A3 in dyslexic children. Mol Psychiatry 2011; 16: 97-107.

31. Dudbridge F, Gusnanto A. Estimation of significance thresholds for genomewide association scans. Genet Epidemiol 2008; 32: 227-234.

32. Purcell S, Neale B, Todd-Brown K, Thomas L, Ferreira MA, Bender D et al. PLINK: a tool set for whole-genome association and population-based linkage analyses. Am J Hum Genet 2007; 81: 559-575.

33. Purcell S, Cherny SS, Sham PC. Genetic power calculator: design of linkage and association genetic mapping studies of complex traits. Bioinformatics 2003; 19: 149-150.

34. Inkster B, Nichols TE, Saemann PG, Auer DP, Holsboer F, Muglia P et al. Association of GSK3beta polymorphisms with brain structural changes in major depressive disorder. Arch Gen Psychiatry 2009; 66: 721-728.

35. Butterworth B. Foundational numerical capacities and the origins of dyscalculia. Trends Cogn Sci 2010; 14: 534-541.

36. Dehaene S. The Number Sense: How the Mind Creates Mathematics. Oxford University Press: New York, 1997.

37. Halberda J, Mazzocco MM, Feigenson L. Individual differences in non-verbal number acuity correlate with maths achievement. Nature 2008; 455: 665-668.

38. Nishioka M, Kohno T, Tani M, Yanaihara N, Tomizawa Y, Otsuka A et al. MYO18B, a candidate tumor suppressor gene at chromosome 22q12.1, deleted, mutated, and methylated in human lung cancer. Proc Natl Acad Sci USA 2002; 99: 12269-12274.

39. Bleeker FE, Lamba S, Rodolfo M, Scarpa A, Leenstra S, Vandertop WP et al. Mutational profiling of cancer candidate genes in glioblastoma, melanoma and pancreatic carcinoma reveals a snapshot of their genomic landscapes. Hum Mutat 2009; 30: E451-E459.

40. Nakano T, Tani M, Nishioka M, Kohno T, Otsuka A, Ohwada S et al. Genetic and epigenetic alterations of the candidate tumor-suppressor gene MYO18B, on chromosome arm 22q, in colorectal cancer. Genes Chromosomes Cancer 2005; 43: 162-171.

41. Ajima R, Akazawa H, Kodama M, Takeshita F, Otsuka A, Kohno T et al. Deficiency of Myo18B in mice results in embryonic lethality with cardiac myofibrillar aberrations. Genes Cells 2008; 13: 987-999.

42. Isogawa $\mathrm{Y}, \mathrm{Kon} \mathrm{T}$, Inoue $\mathrm{T}$, Ohkura $\mathrm{R}$, Yamakawa $\mathrm{H}$, Ohara $\mathrm{O}$ et al. The $\mathrm{N}$-terminal domain of MYO18A has an ATP-insensitive actin-binding site. Biochemistry 2005; 44: 6190-6196.

43. Witke W. The role of profilin complexes in cell motility and other cellular processes. Trends Cell Biol 2004; 14: 461-469.

44. Huang J, Perlis RH, Lee PH, Rush AJ, Fava M, Sachs GS et al. Cross-disorder genomewide analysis of schizophrenia, bipolar disorder, and depression. Am J Psychiatry 2010; 167: 1254-1263.

45. Purcell SM, Wray NR, Stone JL, Visscher PM, O'Donovan MC, Sullivan PF et al. Common polygenic variation contributes to risk of schizophrenia and bipolar disorder. Nature 2009; 460: 748-752.

46. Armstrong E, Schleicher A, Omran H, Curtis M, Zilles K. The ontogeny of human gyrification. Cereb Cortex 1995; 5: 56-63.

47. Rakic P. Specification of cerebral cortical areas. Science 1988; 241: 170-176.

48. Stein JL, Medland SE, Vasquez AA, Hibar DP, Senstad RE, Winkler AM et al. Identification of common variants associated with human hippocampal and intracranial volumes. Nat Genet 2012; 44: 552-561.

Translational Psychiatry is an open-access journal published by Nature Publishing Group. This work is licensed under the Creative Commons Attribution-NonCommercial-No Derivative Works 3.0 Unported License. To view a copy of this license, visit http://creativecommons.org/licenses/by-nc-nd/3.0/

Supplementary Information accompanies the paper on the Translational Psychiatry website (http://www.nature.com/tp) 